\title{
Effect of Baduanjin Exercise Intervention on Cognitive Function and Quality of Life in Women With Breast Cancer Receiving Chemotherapy: Study Protocol of A Randomized Controlled Trial
}

\section{Xiaolin Wei}

Shanghai University of Traditional Chinese Medicine

\section{Ruzhen Yuan}

Shanghai University of Traditional Chinese Medicine

\section{Yongmei Jin}

Seventh People's Hospital Affiliated to Shanghai University of Traditional Chinese Medicine

\section{Shu Li}

Seventh People's Hospital Affiliated to Shanghai University of Traditional Chinese Medicine

\section{Mingyue Wang}

Shanghai University of Traditional Chinese Medicine

\section{Jieting Jiang}

Shanghai University of Traditional Chinese Medicine

Caiqin Wu ( $\sim$ yuwuchina@aliyun.com )

Shanghai university of traditional Chinese medicine https://orcid.org/0000-0002-9420-8958

\section{Kunpeng Li}

Second Rehabilitation Hospital of Shanghai

\section{Study protocol}

Keywords: Baduanjin exercise, Breast cancer, Cognitive function, Quality of life

Posted Date: January 12th, 2021

DOI: https://doi.org/10.21203/rs.3.rs-33100/v1

License: (c) (i) This work is licensed under a Creative Commons Attribution 4.0 International License. Read Full License 


\section{Abstract}

Background: Cancer-related cognitive impairments (CRCl) are frequently reported by cancer patients before and after medical treatment. However, there are no effective interventions to or manage cognitive problem for women with breast cancer. This pilot study was designed to evaluate the protective effect of Baduanjin exercise on cognitive function and cancer-related symptoms in women with early-stage breast cancerundergoing chemotherapy.

Method/design: A single-blinded, randomized controlled trial was designed. The trail will recruit 70 patients with early-stage breast cancer scheduled to receive chemotherapy from Shanghai in China. All participants will be randomized(1:1) to receive either supervised Baduanjin exercise intervention (at least 5 times/week and 30min for each time) plus usual care or usual care alone for three months. The effect of Baduanjin exercise intervention will be evaluated by outcome measures such as subjective and objective cognitive function, symptoms (fatigue, depressive symptoms, anxiety) and health-related quality of life at baseline(T0), 8 weeks(T1) and 12 weeks(T3). Primary cognitive outcomes will be reported descriptively while effect sizes and $95 \%$ confidence intervals (Cls) will be calculated for cognitive measures. The collected data will be analysed using an intention-to-treat principle.

Discussion: This study is the first-known randomized clinical trial to investigate whether Baduanjin exercise plus usual care superior to usual care alone on cognitive function in women with breast cancer receiving chemotherapy. If possible, Baduanjin exercise will be a potential non-pharmacological intervention to manage cognitive function and promote survivorship care among breast cancer survivors.

Trial registration: Chinese Clinical Trial Registry(ChiCTR), ID: ChiCTR 2000033152. Registered on 22 May 2020.

\section{Background}

Cognitive dysfunction is a frequently reported side-effect that cancer patients experienced resulting from cancer and initial medical treatment(eg, surgical resection, chemotherapy regimen, and local radiation therapy, endocrine therapy), showing cognitive decline in the field of memory, processing speed, attention, and executive functions [1-4]. More than $50 \%$ of breast cancer patients have self-reported cognitive problems, and $15-25 \%$ of objective cognitive dysfunction $[5,6]$. Cognitive impairment may persist for years beyond the completion of treatment in some patients [7]. Although the Cancer-related cognitive impairment $(\mathrm{CRCl})$ is mild and/or moderate, it has negative impacts on the physiological condition, activities of daily life, professional achievement[8], treatment adherence[9], even overall quality of life of cancer survivors in the context of long-term cancer care[10,11].

Currently, the critical biological mechanisms of $\mathrm{CRCl}$ are not identified, but there are several have been proposed based on animal and clinical studies by researchers. The mechanisms include: (1) Increase on levels of chemotherapy-induced pro-inflammatory cytokines [12, 13] (e.g. IL-6, TNF-a). (2) Effects of chemotherapy-induced neurotoxicity on brain structure and function (e.g. white matter damage[14], 
inhibition of neurogenesis[15], and changes in neurotransmitter[16]). (3) Changes in central nervous system blood vessels and blood flow[17]. (4) Direct and oxidative DNA damages[18]. In addition, pervasive fatigue and negative emotions may contribute to the susceptibility of decreased cognitive function [19], so it is unclear whether this is the result of a single mechanism or multiple effects.

However, effective interventions are relatively inadequate to alleviate or reduce the risk of cognitive impairment. Various forms of physical activity have been studied to improve cognitive function in healthy older adults and people with mild cognitive impairment (MCI) [20, 21]. Emerging preliminary results demonstrate that physical activities are potential intervention to improve cognitive function, but conflicting results are accumulated. Two pilot studies [22, 23] showed the aerobic exercise improved objective cognition of patients with breast cancer after primary treatment. Hartman et al[22] found processing speed has been improved among breast cancer survivors diagnosed within the past two years after a 12-week exercise intervention, but there was not significant improvement in self-reported cognitive function. Another 24-week supervised and home-based aerobic exercise programme demonstrated similar results in postmenopausal women with early-stage breast cancer [23]. Nevertheless, Galvao et al[24] showed aerobic exercise plus resistance training improved subjective cognitive functioning from a subscale of health-related quality of life scales(EORTC-QLQ-C30) and decreased C-reactive protein(CRP). The conflicting results of previous studies are likely owing to the insufficient samples, heterogeneity of types and timing of exercise interventions delivered. Moreover, most of the studies used self-report and lacked objective multidimensional measures of cognitive function are needed. More rigorous and replicable clinical trials are warranted to prove potential benefits of physical exercise in patients with breast cancer with cognitive problems.

In addition to aerobic exercise, researches based on mind-body therapy have also achieved preliminary results in improving cognitive function for cancer patients[25-27]. Myers et al[25] noted that mindfulness-based exercise might be superior to gentle exercise alone or survivorship support for improving self-reported cognitive function and distress after completion of treatment in survivors with breast cancer. Oh et al[26] demonstrated that a 10-week medical Qigong significantly improved subjective cognition with medium effect size and decreased levels of CRP compared to control group, but the obvious association between CRP levels with self-reported cognition was not observed.

Baduanjin, a traditional Chinese fitness exercise combining meditation and movement with low-intensity, can be tailored to less strenuous formats, more repetitive and easier to study[28, 29], which is considered to be an effective exercise in promoting health[28]. Recently, a review [30] supported that Baduanjin exercise may be effective as an adjunctive rehabilitation method for improving cognitive functions, but the effect of baduanjin exercise on $\mathrm{CRCl}$ are needed to further explore.

Therefore, the purpose of this article is to describe the protocol of a randomized controlled trial to examine whether Baduanjin exercise programme is a protective measure to improve cognitive function in chemotherapy-exposed patients with early-stage breast cancer.

The main aims of the trial are as follows: 
1. Whether 3-month exercise intervention better improves cognitive function during chemotherapy of breast cancer patients in a domain specific fashion such as attention, executive and memory functions.

2. Whether 3-month exercise intervention better improves symptoms and health-related quality of life such as fatigue, depressive symptoms, and anxiety.

3. Whether the association between cognitive complaint or cognitive function and symptoms and health-related quality of life exist.

\section{Methods/design Study design}

This is a single blinded randomized controlled trial (RCT) with two study arms, including a supervised 3month Baduanjin intervention group and a wait-list control group. There will be no follow-up assessments of these participants. Assessors of treatment outcome will be blinded. An overview of the study is displayed in Fig. 1. The overall development of the study protocol followed the SPIRIT (Standard Protocol Items: Recommendations for Interventional Trials) guidelines[31]. The protection of human subject was approved by the Ethics Committee of Seventh People's Hospital of Shanghai University of Traditional Chinese Medicine.

\section{Study setting}

Participants are recruited from the three Affiliated Hospitals of Shanghai University of Traditional Chinese Medicine, including Seventh People's Hospital, Longhua Hospital, and Yueyang Hospital of Integrated Traditional Chinese and Western Medicine respectively. A battery of neuropsychological test and each session are conducted in a private conference room at breast department.

\section{Recruitment}

Recruitment for breast cancer patients started in 1 June 2020. We developed plans and methods to contact patients, and provided the information brochure and study leaflet to help them understand the purpose and program of the trial. Meanwhile, the possible benefits of the exercise programme and the relevant safety during the trial will be explained to them. In addition, we will post recruitment posters in the hospital or via social media such as WeChat, an online APP. According to the inclusion/exclusion criteria, the research team will preliminarily judge potential eligible candidates, and the patients' questionnaire and neuropsychological testing will be assessed and collected, such as measured height and weight to calculate body mass index (BMI) after they have signed the written informed consent.

\section{Inclusion criteria}


Seventy patients with early-stage breast cancer will be recruited. Inclusion criteria meeting this study are:

1. Female patients newly diagnosed stage $₫$ to III breast cancer

2. Schedule to receive adjuvant chemotherapy

3. Aged 40 to 75 years

4. Sufficient fluency of the Chinese language

5. Willing to participate in the study and be randomly assigned.

\section{Exclusion criteria}

The patient will be excluded for meeting any of the following criteria.

1. Disease recurrence or metastasis

2. Known conditions and/or diseases that impact cognition (eg, Alzheimer's disease, dementia, or other psychological diagnoses)

3. Disorders that might preclude exercise participation (eg, fracture, arthritis)

4. Current/planned participation in mindfulness-based exercise programs (eg, TaiChi or Qigong).

\section{Randomization and blinding}

Participants were randomly assigned to 1 of 2 groups-an intervention group or a control group-in a 1:1 ratio using SPSS 24.0 software. The allocation of the result will be put into sealed, opaque envelopes by a study administrator not involved in the study and the group numbers will be subsequently disclosed. Because baduanjin exercise is a well-known exercise for the Chinese people, blinding is conducted to assessors only, not physicians and patients. Apart from this, all participants will be asked not to discuss with one another their practice in the trial to minimize biases as much as possible. The assessor will be also instructed not to acquire participant's exercise information. Participants are allowed to withdraw without giving a reason at any time but the withdrawal of patient need to be determined by physician and the patient in the process.

\section{Interventions}

Upon completion of baseline testing and randomization, participants are notified of group assignment by the unblined project director. At that time, a participant's group assignment, exercise or usual care, is scheduled to begin within 2 weeks of beginning chemotherapy.

\section{Baduanjin group}


Baduanjin exercise intervention is conducted according to the standards promulgated by the General Administration of Sport in China in 2003, which consists of 10 postures (including the beginning and ending posture). The professionally trained Qigong personnel will teach Baduanjin exercises to subject researchers, and then the researchers conduct on-site instruction, guide patients, and correct their movements. The relevant video will be distributed to the patients. Exercise methods include group training and home-based exercise. The patients will be trained by researchers in the recreation for patient during the first chemotherapy for one week in the hospital, then practice at home followed the video. Recommended training time was five weekly 30 -min each time for 12 weeks, and it is advisable not to feel tired. During the process of exercise, the exercise will start after stretching the joints and inhalation and exhalation for 2 minutes respectively, and practice Baduanjin twice for 12 minutes each time, then eventually followed by muscle relaxation exercises for $2 \mathrm{~min}$. Sessions may be held in small groups in the first week. Patients' log will be recorded including any obstacles during exercise at home. When the patients go to the hospital to receive chemotherapy, the feedback of family exercise log will be collected. At the same time, the researchers will track and record the training situation through established online WeChat group.

\section{Wait-list group}

Patients randomized to the control group are provided oral and brochures to maintain their usual healthy lifestyle according to the guideline and standard for the diagnosis and treatment of breast cancer by the Chinese Anti-Cancer Association (2019 edition). Disease-related questions raised by the subjects will be directly communicated or answered on WeChat online. The patient's limb affected can perform abduction, forward flexion, backward extension, internal rotation, and wall climbing according to the recommendation of the therapist, the patients are not asked to conduct other aerobic activities. The supervised Baduanjin exercise for patients will be offered if they want after the completion of study.

\section{Study assessments}

At pre-intervention (T0), week 8(T1) and week 12(T2) during intervention, participants complete all outcome assessment by professional researchers. General characteristics information needs to be collected at baseline, including patients' age, level of education and economic status via interview and retrieved disease information from medical records by two trained study coordinators (M-YW, J-TJ). The schedule of study outcome assessments is outlined in Fig. 2.

\section{Cognitive ability}

The International Cognition and Cancer Task Force (ICCTF)[1] proposed and considered that objective neuropsychological test battery is the gold standard for evaluating cognitive function of $\mathrm{CRCl}$. The Hopkins Verbal Learning Test-Revised (HVLT-R)[32] used is a test to assess verbal learning and memory. 
The HVLT Chinese version consists of a 12-word list, and the researcher will read them aloud. The patient needs to recall as many words as possible for three consecutive learning trials (total recall). After 20 min, the patient is asked again to recall (delayed recall). Lastly, a list of 24 words is read by the researcher and the patient is asked to declare and answer whether the concerned word was mentioned in previous list (recognition). At the next follow-up, a parallel version of HVLT (the 12-word list with different words) is applied to replace the original version. In addition, executive function and attention are tested by TrailMaking Test (TMT)[33] and Digit Symbol Substitution Test (DSST), respectively (see Table 1).

In addition, patients' subjective feelings are the most relevant measures of clinical significance, and cognitive complaint reported by patients is the main outcome indicator. The Functional Assessment of Cancer Therapy-Cognitive Function (FACT-Cog) developed by Wagner et al[34] was to assess subjective cognitive functioning, and revised Chinese version will be employ in this study[35]. Cronbach's a coefficient is from 0.87 to 0.96 with good internal consistency. The scale was divided into 4 dimensions including 37 items: perceived cognitive impairment $(\mathrm{PCl})$, perceived cognitive ability (PCA), evaluation of others, and impact on quality of life (QoL). The higher score of the overall cognitive impairment indicated the better the cognitive function. The FACT-Cog subscales in this study will be utilized including PCI, PCA, QoL.

\section{Patient-reported outcomes}

Patients will complete several patient-reported outcomes (PRO) questionnaires on subjective fatigue, anxiety, depression, and health-related quality of life (see Table 2).

\section{Safety}

Adverse events occurred in the study should be truthfully recorded in the adverse event record form, including its symptoms, occurrence time, duration, treatment measures, etc. Adverse events were divided into general adverse events, major adverse events and serious adverse events according to their severity. A report form should be completed for a serious adverse event (SAE). Comprehensive factors, security evaluation. Evaluation parameters: level 1 (safety, no adverse reactions); Level 2 (relatively safe, mild adverse reactions, no need to do any treatment, can continue training); Level 3 (safety problems, moderate adverse reactions, treatment can be continued training); Level 4 (study should be suspended due to serious adverse reactions). According to the actual situation, after the end of the intervention, the safety of the subjects during the experiment will be evaluated. An adverse event that meets the criteria for SAE between study enrollment and hospital discharge will be reported to the local Institutional Review Board.

\section{Trial monitoring}


The trial is at a low risk without stopping rules, so Investigational Medicinal Products will not be used. The independent Trial Oversight Committee (TOC) will review the protocol and the statistical analysis plan, and review recruitment of patients, compliance with protocol, adverse events or effects, and the collection of complete data. The research staff are independent experts specialising in clinical trials, cancer nursing, or statistics. The Trial Management Committee will supervise trial procedures and progress, and the outcomes will be reported regularly.

\section{Protocol amendments}

The sponsor is responsible for submitting materials to the local ethics committee.During this process, the principal investigator may be trained in any programme modification and, if necessary, any retraining shall be provided.

\section{Sample size}

The sample size was estimated in this study by using perceptive cognitive impairment $(\mathrm{PCl})$ as the main effect indicator, which refers to the results of Oh et al[26]. Using $G$ * power 3.1 software, 58 cases of the total sample can be calcutated with a test power of 0.80 . Considering the $20 \%$ dropout rate, the total sample size required for this study is about 70 patients randomly randomized to the intervention group or the usual care group.

\section{Data Collection}

All the data will be collected from these participants, including those who discontinue treatment. Most of the outcome data will be collected via the provided paper-and-pencil measures (anonymised by participant ID). If participants interest in electronic measures (anonymised by participant ID), these will be provided.

The cognitive assessors will conduct a comprehensive training in scoring the measures. Interrater reliability will be determined, and discrepancies will be resolved by consensus.

When the participants have been randomized, dropout and premature termination from two groups at any point will be recorded along with revalant reason. Participants can choose to withdraw and will not be affected at any time while declining to participate or withdrawing from the trial. A participant may be withdrawn from the intervention in some cases. For example, they develop a condition which would exclude them from the study based on the eligibility criteria. Participants who are withdrawn from the intervention during the trial will not continue in follow-up but will not be replaced.

\section{Data management}


The data are collected by the research staff and will be stored in a database. The identifiable information (eg. real name) of the participants will not appear in the relevant reports of the trial to protect their privacy. Only researchers directly involved in the analysis of the RCT will have access to the final trial data set, which will only contain coded data. After data collection and before data storage, all obtained outcomes are manually double-checked by the research staff (M-YW, J-TJ). The design, intervention process, study integrity and safety aspects will be monitored at group meetings involved in this study.

\section{Data analysis}

Demographics and other characteristics will be reported descriptively to summarize the distribution of all variables via statistics software (SPSS 24.0). Means and SD will be calculated for continuous normally distributed variables and medians and ranges for non-normally distributed variables. Categorical variables will be presented by absolute numbers and percentages.

For the primary intention-to-treat (ITT) analysis, we will compare the number of patients showing postintervention improvement in the HVLT-R between the intervention group and control group using Fisher's exact tests. The treatment effect will be expressed as an estimate with $95 \% \mathrm{Cls}$, adjusted for key prognostic factors (eg, age, level of education) and baseline HVLT-R, estimated from log-binomial regression models. Analysis of covariance (ANCOVA) will be used to analyse the ITT effect on other neuropsychological outcome measures. All cases for both baseline and follow-up measurements will be analysed for the different outcomes. If missing values exceed $10 \%$, we will impute missing values on covariates by using multiple imputation.

Adherence to the Baduanjin exercise will be noted (the number of attended sessions divided by the number of sessions offered). Linear regression analysis adjusted for baseline will be used to assess the association between changes in serum levels of inflammatory factors and changes in cognitive functioning. The same analyses will be conducted for the association between changes in cognitive functioning and changes in quality of life.

For the long-term effects, a liner mixed-effect model will be used to analyze the effects of the baseline measurements and two follow-up measurements at 8 and 12 weeks simultaneously. In addition, reliable change indices (RCls) for all individual patients were calculated for changes in all neuropsychological assessment raw scores. Individual change was defined by $\mathrm{RCl}$ values exceeding \pm 1.645 (corresponding with a $90 \% \mathrm{Cl}$ ). The numbers of patients with reliably improved, declined, or non-changed performance was compared between groups for each neuropsychological test variable. Two-sided significance level for all tests is 0.05 .

\section{Dissemination}


The findings of this study will be reported in a master's thesis by the main author, will be submitted to a peer-reviewed journal for publication, and will be presented at relevant conferences on the subject of matter.

\section{Discussion}

Currently, although cognitive decline is a well-known phenomenon, there are no pharmacological interventions to prevent or alleviate cognitive problems in patients with various non-central nervous system cancers. Physical exercise has been recommended as part of rehabilitation, which appears to a feasible and promising non-drug intervention that improves cognitive abilities. Preclinical study[36] have demonstrated that low-intensity exercise may assist in preventing cognitive dysfunction in chemotherapyexposed patients with breast cancer. Bduanjin exercise as a mind-body therapy has beneficial effects on fatigue, quality of life, negative mood among patients $[30,37,38]$. In addition, this training exercise is easy to learn and widely used in the community with no restriction for the environment[39]. Some evidences have demonstrated that improved the attention of the elderly or patients with MC[40, 41]. In light of the previous state of the literature, we hypothesized that the exercise programme may have a protective effect on the cognitive function of breast cancer patients. Given ethical reasons, patients assigned into the control arm have the opportunity to carry out a 3-month exercise programme after the study period, so as to decrease subsequently the risk of sample contamination. If Baduanjin exercise seems to be effective, it can provide evidence-based interventions during cancer treatment to possibly minimize or decrease cognitive problems, thereby improving their quality of life. Previous preclinical and human studies have shown that a wider range of chemotherapy is associated with cognitive changes in cancer patients, so we hope that the results of this study will also be applicable to patients with other types of tumors and receiving chemotherapy. Therefore, the ultimate purpose of this study may have a positive impact on the quality of life or physical and mental health of growing survivors with cancer.

\section{Trial Status}

Recruitment of patients started on 1 June 2020. The trial is currently underway and expected to be completed by 1 June 2021. The protocol was 1.0 version. Date of edition was May 22, 2020.

\section{Abbreviations}

HVLT-R: Hopkins Verbal Learning Test-Revised; FACT-Cog: Functional Assessment of Cancer TherapyCognitive Function; PCl: Perceived Cognitive Impairment; PCA: Perceived Cognitive Ability; TMT: TrailMaking Test; DSST: Digit Symbol Substitution Test; MFI: Multidimensional Fatigue Inventory; HADS: Hospital Anxiety and Depression Scale; FACT-B: Functional Assessment of Cancer Therapy-Breast; RCls: Reliable Change Indices; TOC: Trial Oversight Committee; SAE: Serious Adverse Event

\section{Declarations}


Acknowledgements

We gratefully acknowledge all participants who contribute to the trial.

\section{Author contributions}

C-QW and K-PL conceived and designed this research and supervised the research team. X-LW and R-ZY were the main implementers of the study and drafted manuscripts. Y-MJ and SL wrote the ethical review confirmation. M-YW and J-TJ participated in the design of the study and assisted in drafting the manuscript. All authors have read and agreed the final version of the manuscript.

\section{Funding}

The trail was supported by the Nursing Subject Competence Enhancement Project of Shanghai University of Traditional Chinese Medicine (No.2020HLXK07) and Xinglin Youth Talents Training Project of Shanghai University of Traditional Chinese Medicine-Xinglin Scholar to Cai-Qin Wu. The funder plays no role in the design of study, data collection and analysis, or preparation of the manuscript.

\section{Availability of data and material}

Data sharing is not applicable to this trial as no database is generated or analyzed for the current study.

\section{Ethics approval and consent to participate}

This study was approved on 12 May by the Ethics Committee of Seventh People's Hospital affiliated to Shanghai University of Traditional Chinese Medicine (referrence number 2020-7th-HIRB-011). All participants will sign informed consent, consent to initial assessent and participate in this study.

\section{Consent for publication}

No applicable.

\section{Competing interests}

The authors declare that they have no competing interests.

\section{Author details}

${ }^{1}$ School of Nursing, Shanghai University of Traditional Chinese Medicine, shanghai, China. ${ }^{2}$ Department of Nursing, the Seventh People's Hospital Affiliated to Shanghai University of Traditional Chinese Medicine, 358 Datong Road, Shanghai, 200137, China. ${ }^{3}$ Department of Respiratory Medicine, the Seventh People's Hospital Affiliated to Shanghai University of Traditional Chinese Medicine, 358 Datong Road, Shanghai, 200137, China. ${ }^{4}$ Department of Neurology, the Second Rehabilitation Hospital of Shanghai, 860 Changjiang Road, Shanghai, 200441. 


\section{References}

1. Wefel JS, Vardy J, Ahles T, Schagen SB. International Cognition and Cancer Task Force recommendations to harmonise studies of cognitive function in patients with cancer. Lancet Oncol. 2011;12(7):703-8. doi:10.1016/S1470-2045(10)70294-1.

2. Deprez S, Kesler SR, Saykin AJ, Silverman DHS, de Ruiter MB, McDonald BC. International Cognition and Cancer Task Force Recommendations for Neuroimaging Methods in the Study of Cognitive Impairment in Non-CNS Cancer Patients. J Natl Cancer Inst. 2018;110(3):223-31. doi:10.1093/jnci/djx285.

3. Wefel JS, Kesler SR, Noll KR, Schagen SB. Clinical characteristics, pathophysiology, and management of noncentral nervous system cancer-related cognitive impairment in adults. CA Cancer J Clin. 2015;65(2):123-38. doi:10.3322/caac.21258.

4. Lange M, Joly F, Vardy J, Ahles T, Dubois M, Tron L, et al. Cancer-related cognitive impairment: an update on state of the art, detection, and management strategies in cancer survivors. Ann Oncol. 2019;30(12):1925-40. doi:10.1093/annonc/mdz410.

5. Ahles TA, Root JC, Ryan EL. Cancer- and cancer treatment-associated cognitive change: an update on the state of the science. J Clin Oncol. 2012;30(30):3675-86. doi:10.1200/jco.2012.43.0116.

6. Lange M, Licaj I, Clarisse B, Humbert X, Grellard JM, Tron L, et al. Cognitive complaints in cancer survivors and expectations for support: Results from a web-based survey. Cancer medicine. 2019;8(5):2654-63. doi:10.1002/cam4.2069.

7. Koppelmans V, Breteler MM, Boogerd W, Seynaeve C, Gundy C, Schagen SB. Neuropsychological performance in survivors of breast cancer more than 20 years after adjuvant chemotherapy. $\mathrm{J}$ Clin Oncol. 2012;30(10):1080-6. doi:10.1200/jco.2011.37.0189.

8. Boykoff N, Moieni M, Subramanian SK. Confronting chemobrain: an in-depth look at survivors' reports of impact on work, social networks, and health care response. J Cancer Surviv. 2009;3(4):223-32. doi:10.1007/s11764-009-0098-x.

9. Camacho FT, Tan X, Alcalá HE, Shah S, Anderson RT, Balkrishnan R. Impact of patient race and geographical factors on initiation and adherence to adjuvant endocrine therapy in medicare breast cancer survivors. Medicine. 2017;96(24):e7147. doi:10.1097/md.0000000000007147.

10. Bender CM, Merriman JD. Cancer- and treatment-related cognitive changes: what can we do now? What lies ahead? Oncology. (Williston Park NY). 2014;28(9):806-8.

11. Von Ah D, Habermann B, Carpenter JS, Schneider BL. Impact of perceived cognitive impairment in breast cancer survivors. European journal of oncology nursing: the official journal of European Oncology Nursing Society. 2013;17(2):236-41. doi:10.1016/j.ejon.2012.06.002.

12. Lyon DE, Cohen R, Chen H, Kelly DL, McCain NL, Starkweather A, et al. Relationship of systemic cytokine concentrations to cognitive function over two years in women with early stage breast cancer. J Neuroimmunol. 2016;301:74-82. doi:10.1016/j.jneuroim.2016.11.002. 
13. Pomykala KL, Ganz PA, Bower JE, Kwan L, Castellon SA, Mallam S, et al. The association between pro-inflammatory cytokines, regional cerebral metabolism, and cognitive complaints following adjuvant chemotherapy for breast cancer. Brain imaging behavior. 2013;7(4):511-23. doi:10.1007/s11682-013-9243-2.

14. Stouten-Kemperman MM, de Ruiter MB, Caan MW, Boogerd W, Kerst MJ, Reneman L, et al. Lower cognitive performance and white matter changes in testicular cancer survivors 10 years after chemotherapy. Hum Brain Mapp. 2015;36(11):4638-47. doi:10.1002/hbm.22942.

15. Lazarov O, Mattson MP, Peterson DA, Pimplikar SW, van Praag H. When neurogenesis encounters aging and disease. Trends Neurosci. 2010;33(12):569-79. doi:10.1016/j.tins.2010.09.003.

16. Lojovich JM. The relationship between aerobic exercise and cognition: is movement medicinal? J Head Trauma Rehabil. 2010;25(3):184-92. doi:10.1097/HTR.0b013e3181dc78cd.

17. PN A, JD C, KP G, C SL. M, R S, et al. Elevation in cerebral blood flow velocity with aerobic fitness throughout healthy human ageing. 2008;586(16):4005-10. doi: 10.1113/jphysiol.2008.158279.

18. Ahles TA, Saykin AJ. Candidate mechanisms for chemotherapy-induced cognitive changes. Nature reviews Cancer. 2007;7(3):192-201. doi:10.1038/nrc2073.

19. Hampson JP, Zick SM, Khabir T, Wright BD, Harris RE. Altered resting brain connectivity in persistent cancer related fatigue. Neurolmage Clinical. 2015;8:305-13. doi:10.1016/j.nicl.2015.04.022.

20. Baker LD, Frank LL, Foster-Schubert K, Green PS, Wilkinson CW, McTiernan A, et al. Aerobic exercise improves cognition for older adults with glucose intolerance, a risk factor for Alzheimer's disease. J Alzheimers Dis. 2010;22(2):569-79. doi:10.3233/JAD-2010-100768.

21. Baker LD, Frank LL, Foster-Schubert K, Green PS, Wilkinson CW, McTiernan A, et al. Effects of aerobic exercise on mild cognitive impairment: a controlled trial. Arch Neurol. 2010;67(1):71-9. doi:10.1001/archneurol.2009.307.

22. Hartman SJ, Nelson SH, Myers E, Natarajan L, Sears DD, Palmer BW, et al. Randomized controlled trial of increasing physical activity on objectively measured and self-reported cognitive functioning among breast cancer survivors: The memory \& motion study. Cancer. 2018;124(1):192-202. doi:10.1002/cncr.30987.

23. Campbell KL, Kam JWY, Neil-Sztramko SE, Liu Ambrose T, Handy TC, Lim HJ, et al. Effect of aerobic exercise on cancer-associated cognitive impairment: A proof-of-concept RCT. Psychooncology. 2018;27(1):53-60. doi:10.1002/pon.4370.

24. Galvão DA, Taaffe DR, Spry N, Joseph D, Newton RU. Combined resistance and aerobic exercise program reverses muscle loss in men undergoing androgen suppression therapy for prostate cancer without bone metastases: a randomized controlled trial. J Clin Oncol. 2010;28(2):340-7. doi:10.1200/JCO.2009.23.2488.

25. Myers JS, Mitchell M, Krigel S, Steinhoff A, Boyce-White A, Van Goethem K, et al. Qigong intervention for breast cancer survivors with complaints of decreased cognitive function. Support Care Cancer. 2019;27(4):1395-403. doi:10.1007/s00520-018-4430-8. 
26. Oh B, Butow PN, Mullan BA, Clarke SJ, Beale PJ, Pavlakis N, et al. Effect of medical Qigong on cognitive function, quality of life, and a biomarker of inflammation in cancer patients: a randomized controlled trial. Support Care Cancer. 2012;20(6):1235-42. doi:10.1007/s00520-011-1209-6.

27. Janelsins MC, Peppone LJ, Heckler CE, Kesler SR, Sprod LK, Atkins J, et al. YOCAS@ $®$ Yoga Reduces Self-reported Memory Difficulty in Cancer Survivors in a Nationwide Randomized Clinical Trial: Investigating Relationships Between Memory and Sleep. Integrative Cancer Therapies. 2015;15(3):263-71. doi:10.1177/1534735415617021.

28. Kelley GA, Kelley KS. Meditative Movement Therapies and Health-Related Quality-of-Life in Adults: A Systematic Review of Meta-Analyses. PLoS One. 2015;10(6):e0129181. doi:10.1371/journal.pone.0129181.

29. Larkey LK, Roe DJ, Weihs KL, Jahnke R, Lopez AM, Rogers CE, et al. Randomized controlled trial of Qigong/Tai Chi Easy on cancer-related fatigue in breast cancer survivors. Annals of behavioral medicine: a publication of the Society of Behavioral Medicine. 2015;49(2):165-76. doi:10.1007/s12160-014-9645-4.

30. Zou L, Pan Z, Yeung A, Talwar S, Wang C, Liu Y, et al. A Review Study on the Beneficial Effects of Baduanjin. Journal of alternative complementary medicine (New York NY). 2018;24(4):324-35. doi:10.1089/acm.2017.0241.

31. AW C, JM T, DG A, PC AL, K K-J G, et al. SPIRIT 2013 statement: defining standard protocol items for clinical trials. Ann Intern Med. 2013;158(3):200-7. doi:10.7326/0003-4819-158-3-201302050-00583.

32. Shi J, Tian J, Wei M, Miao Y, Wang Y. The utility of the Hopkins Verbal Learning Test (Chinese version) for screening dementia and mild cognitive impairment in a Chinese population. BMC Neurol. 2012;12:136. doi:10.1186/1471-2377-12-136.

33. Wei M, Shi J, Li T, Ni J, Zhang X, Li Y, et al. Diagnostic Accuracy of the Chinese Version of the TrailMaking Test for Screening Cognitive Impairment. J Am Geriatr Soc. 2018;66(1):92-9. doi:10.1111/jgs.15135.

34. Wagner LI, Sweet J, Butt Z, Lai JS, Cella D. Measuring patient self-reported cognitive function: Development of the Functional Assessment of Cancer Therapy-Cognitive Function Instrument. $J$ Support Oncol. 2009;7:W32-W9.

35. Cheung YT, Lim SR, Shwe M, Tan YP, Chan A. Psychometric properties and measurement equivalence of the English and Chinese versions of the functional assessment of cancer therapy-cognitive in Asian patients with breast cancer. Value in health: the journal of the International Society for Pharmacoeconomics Outcomes Research. 2013;16(6):1001-13. doi:10.1016/j.jval.2013.06.017.

36. Park HS, Kim CJ, Kwak HB, No MH, Heo JW, Kim TW. Physical exercise prevents cognitive impairment by enhancing hippocampal neuroplasticity and mitochondrial function in doxorubicininduced chemobrain. Neuropharmacology. 2018;133:451-61. doi:10.1016/j.neuropharm.2018.02.013.

37. Lu Y, Qu HQ, Chen FY, Li XT, Cai L, Chen S, et al. Effect of Baduanjin Qigong Exercise on CancerRelated Fatigue in Patients with Colorectal Cancer Undergoing Chemotherapy: A Randomized 
Controlled Trial. Oncology research treatment. 2019;42(9):431-9. doi:10.1159/000501127.

38. Ying W, Min QW, Lei T, Na ZX, Li L, Jing L. The health effects of Baduanjin exercise (a type of Qigong exercise) in breast cancer survivors: A randomized, controlled, single-blinded trial. European journal of oncology nursing: the official journal of European Oncology Nursing Society. 2019;39:90-7. doi:10.1016/j.ejon.2019.01.007.

39. Antonishen K. Exercise mode heterogeneity among reported studies of the qigong practice Baduanjin. J Bodyw Mov Ther. 2015;19(2):278-83. doi:10.1016/j.jbmt.2014.05.013.

40. $10.3389 /$ fpsyg. 2019.02075

Xia R, Qiu P, Lin H, Ye B, Wan M, Li M, et al. The Effect of Traditional Chinese Mind-Body Exercise (Baduanjin) and Brisk Walking on the Dorsal Attention Network in Older Adults With Mild Cognitive Impairment. Frontiers in psychology. 2019;10:2075. doi: 10.3389/fpsyg.2019.02075.

41. Tao J, Liu J, Chen X, Xia R, Li M, Huang M, et al. Mind-body exercise improves cognitive function and modulates the function and structure of the hippocampus and anterior cingulate cortex in patients with mild cognitive impairment. Neurolmage Clinical. 2019;23:101834.

doi:10.1016/j.nicl.2019.101834.

\section{Tables}

Due to technical limitations, table 1 and 2 is only available as a download in the Supplemental Files section.

\section{Figures}




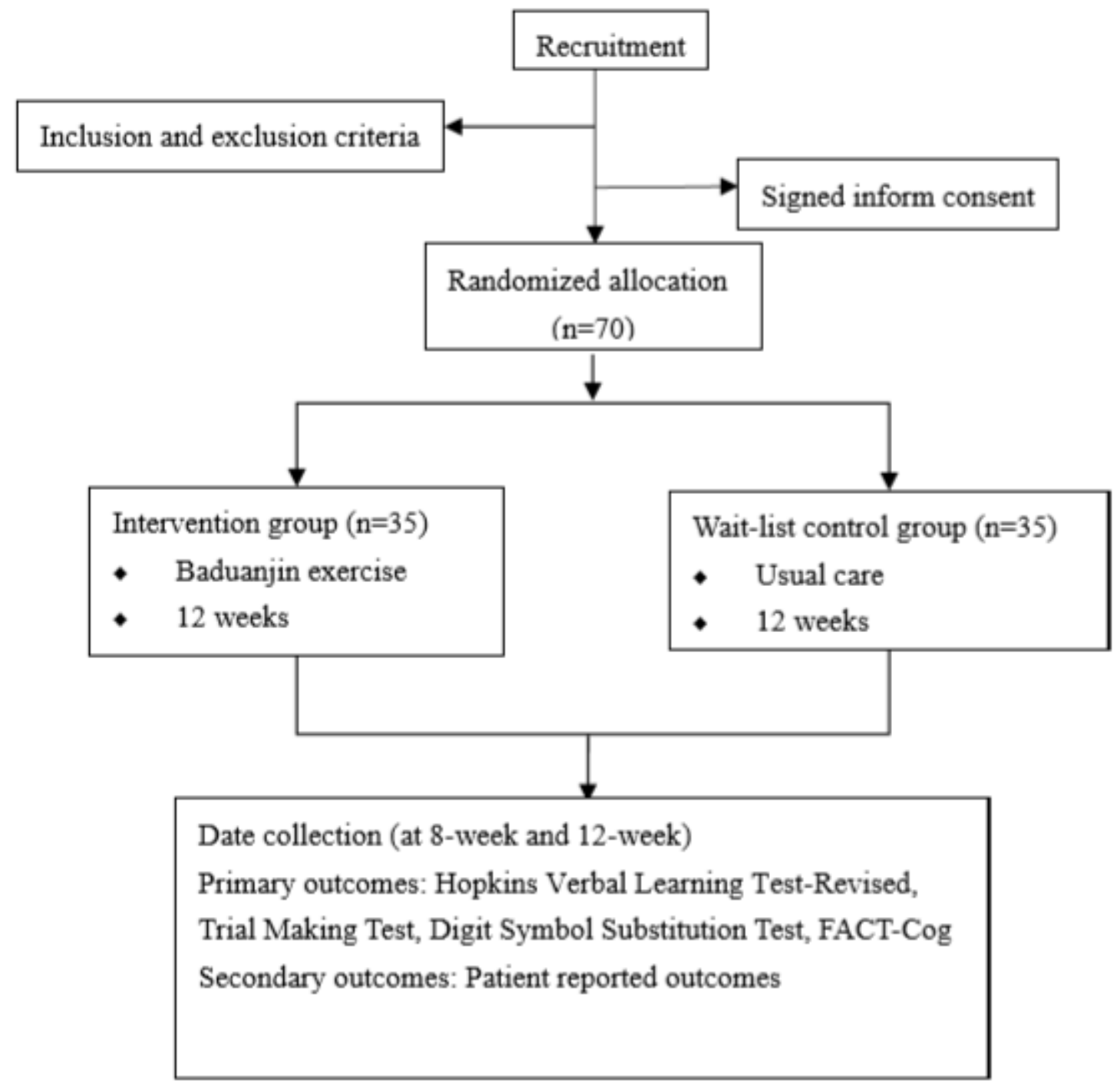

Figure 1

Overview of the study 


\begin{tabular}{|c|c|c|c|c|c|c|}
\hline & Enrolment & $\begin{array}{l}\text { Baseline } \\
\text { assessment }\end{array}$ & Allocation & Intervention & $\begin{array}{l}\text { Intervention } \\
8 \\
\text { weeks(T1) }\end{array}$ & $\begin{array}{l}\text { Intervention } \\
12 \\
\text { weeks(T2) }\end{array}$ \\
\hline TIMEPOINT & & T0 & & & & \\
\hline \multicolumn{7}{|l|}{ ENROLMENT: } \\
\hline Eligibility screen & $\mathrm{X}$ & & & & & \\
\hline Informed consent & $\mathrm{X}$ & & & & & \\
\hline \multicolumn{7}{|l|}{ RANDOMISATION: } \\
\hline Allocation & & & $\mathrm{X}$ & & & \\
\hline \multicolumn{7}{|l|}{ INTERVENTIONS: } \\
\hline \multicolumn{7}{|l|}{$\begin{array}{r}\text { Baduanjin exercise } \\
\text { intervention }\end{array}$} \\
\hline Wait-list control & & & & & & $\longrightarrow$ \\
\hline \multicolumn{7}{|l|}{ ASSESSMENTS: } \\
\hline $\begin{array}{l}\text { Retrospective } \\
\text { questionnaire }\end{array}$ & & $\mathrm{X}$ & & & & \\
\hline Neurocognitive testing & & $\mathrm{X}$ & & & $\mathrm{X}$ & $\mathrm{X}$ \\
\hline $\begin{array}{r}\text { Subjective cognitive } \\
\text { complaint }\end{array}$ & & $\mathrm{X}$ & & & $\mathrm{X}$ & $\mathrm{X}$ \\
\hline $\begin{array}{r}\text { Cancer-related } \\
\text { symptoms }\end{array}$ & & $\mathrm{X}$ & & & $\mathrm{X}$ & $\mathrm{X}$ \\
\hline $\begin{array}{r}\text { Adverse effects } \\
\text { or events } \\
\end{array}$ & & & & & $\mathrm{X}$ & $\mathrm{X}$ \\
\hline Adherence to exercise & & & & & $\mathrm{X}$ & $\mathrm{X}$ \\
\hline
\end{tabular}

Figure 2

Standard Protocal Items: Recommendations for Interventional Trials(SPIRIT) diagram of enrolment, treatment, and assessments over intervention time

\section{Supplementary Files}

This is a list of supplementary files associated with this preprint. Click to download.

- SPIRIT2013checklist.doc

- Table.pdf 